\title{
Heart disease mortality following bereavement - the OPCS longitudinal study (abstract)
}

\author{
D.R. Jones \\ Department of Clinical Epidemiology, St. George's Hospital Medical School, London SW17 ORE, UK.
}

Many studies have suggested that following the experience of stressful life events the risks of myocardial infarction, accidents and perhaps other diseases are elevated. In the OPCS Longitudinal Study routinely collected data on deaths, and deaths of a spouse occurring in a $1 \%$ sample of the population of England and Wales in the period 1971-81 are linked together, and with 1971 Census records of sample members. The timing and patterns of death following the potentially very stressful event of conjugal bereavement may thus be analysed.

Overall, the mortality (from ischaemic heart disease) was less than $10 \%$ in excess of that in all members of the LS sample. As in many earlier studies, some increase in death rates shortly after widow(er)hood are observed. Unusually, for deaths from all causes these increases are more marked in widows than in widowers with, for example, a two-fold increase in mortality from all causes in the first month after widowhood. However, no peak of post-bereavement mortality from ischaemic heart disease is clearly established in either sex.

Although the study is large, with a well-chosen control group, only a limited characterization of study members from data collected in the Census is possible: in particular, no measures of personality, behaviour or diet are available. Gradients in post-bereavement mortality similar to those in general mortality were found with several measures of socio-economic status, including social class. Investigation of potential effects of social or familial support, as measured by household structure and numbers of children, led to equivocal results.

Several possible explanations for the increased mortality rates are examined. Hypotheses based on common marital environment, homogamy or simultaneous accidental death are seen to be of very limited value. The observed patterns, although consistent with an early effect of a stressful life event, do not suggest that stress following bereavement leads to an excess of ischaemic heart disease mortality.

Correspondence: D.R. Jones, Ph.D. 